\title{
Enhanced recovery after rectal surgery: what we have learned so far
}

\author{
Giovanni D. Tebala, Ayeshah Gordon-Dixon, Mohammad Imtiaz, Ashish Shrestha, Mohamed Toeima \\ Colorectal and Emergency Surgery Unit, East Kent Hospitals University NHS Foundation Trust, William Harvey Hospital, \\ Ashford, Kent TN24 OLZ, UK.
}

Correspondence to: Giovanni D. Tebala, Colorectal and Emergency Surgery Unit, East Kent Hospitals University NHS Foundation Trust, William Harvey Hospital, Kennington Rd, Willesborough, Ashford, Kent TN24 OLZ, UK.

E-mail: giovanni.tebala@nhs.net

How to cite this article: Tebala GD, Gordon-Dixon A, Imtiaz M, Shrestha A, Toeima M. Enhanced recovery after rectal surgery: what we have learned so far. Mini-invasive Surg 2018;2:32. http://dx.doi.org/10.20517/2574-1225.2018.37

Received: 8 Jun 2018 First Decision: 27 Aug 2018 Revised: 31 Aug 2018 Accepted: 3 Sep 2018 Published: 28 Sep 2018

Science Editor: Gordon N. Buchanan Copy Editor: Cai-Hong Wang Production Editor: Zhong-Yu Guo

\begin{abstract}
Enhanced recovery after surgery (ERAS) protocols are gradually becoming the gold standard in the perioperative management of rectal patients. It is a multimodal and multidisciplinary approach that has the great merit to involve and empower the patient and bring him or her back to the centre of the strategy of care. If applied correctly, ERAS can improve the postoperative recovery, reduce the rate of complications and reduce the postoperative length of stay, in patients who had extensive pelvic dissection. The factors within ERAS and their application do not represent rigid schematizations but fluid concepts that may undergo substantial changes as soon as new evidence becomes available. The ERAS principles must be adapted to the specific environment and each team is expected to set up their own programme and quality control criteria. In this comprehensive review, the latest evidence and trend on enhanced recovery after rectal surgery have been critically appraised and presented.
\end{abstract}

Keywords: Laparoscopic surgery, rectal surgery, enhanced recovery after surgery

\section{INTRODUCTION}

The enhanced recovery after surgery (ERAS) programmes have been introduced in the 90s to improve the outcome of patients undergoing major surgery ${ }^{[1,2]}$. The concept at the basis of ERAS is to apply the best and most recent evidences of the literature to set up standardized perioperative protocols to improve the postoperative recovery and, only as an obvious consequence, to reduce the length of hospital stay (LOS) ${ }^{[3-5]}$. Unfortunately, the name initially used for these new protocols - "Fast Track" - sounded like a suggestion

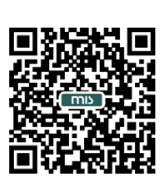


to discharge surgical patients too early and probably unsafely and many patients regarded it only as a way to reduce the costs for the hospital, without any benefit for them who, on the contrary, were exposed to serious risks at home for being discharged prematurely. At the same time, many doctors were concerned regarding the possible medico-legal risks, if complications occurred in patients after their early discharge.

On the contrary, the more recent and positive denomination of ERAS evokes a significant improvement of the experience of the patient undergoing major surgery. ERAS protocols aim at optimising the postoperative recovery as they "reduce surgical stress, maintain postoperative physiological function, and enhance mobilization after surgery, ${ }^{\text {,[6] }}$.

In fact, it has been demonstrated that elective patients treated with ER protocols recover better and quicker with respect to those treated under the traditional protocols. Time to first flatus, time to full oral intake and time to full mobilisation are reduced within an ERAS protocol with respect to the traditional postoperative care, and this leads to improvements in patient satisfaction and reduction of costs ${ }^{[4]}$.

General morbidity and specific medical complication rates after surgery are reduced, although the rate of surgical complications doesn't seem to be affected ${ }^{[7-11]}$. On a physiological level, the control of insulinresistance index and the reduction of the levels of cortisol and cytokines with respect to the "traditional" management demonstrate that ERAS yields a reduction of the postoperative stress response and hence the risk of complications ${ }^{[12]}$.

As a consequence, postoperative LOS is reduced as well ${ }^{[12,13]}$. The UK national audit results published in 2015 confirmed that adherence to ERAS pathway is weakly but significantly associated with overall reduction of LOS. The weakness of this association may raise the hypothesis that it is more than single elements of ERAS acting directly on LOS and that ERAS as a whole, yields a mentality change that leads to better postoperative recovery $^{[14]}$, also in very elderly patients ${ }^{[15]}$.

Instead of increasing the risk of complications, as initially feared, early discharge seems to be a protective factor towards complications ${ }^{[12]}$. Although we feel this may be considered an over optimistic message, it is obvious that discharging a patient early in their postoperative course would allow him or her to recover completely in their own environment, thus decreasing the risk of hospital-acquired infection and other complications linked to prolonged hospitalisation, including psychological issues.

Clearly, it is important that every system is in place to make sure that patients are adequately followed up at home in their early postoperative period, even if by phone only, and they have a clear pathway to access senior review if and when needed.

Furthermore, ERAS allows an early warning for eventual complications. In fact, a patient who does not recover as expected in the postoperative period - delayed recovery of their bowel function, altered vital signs and parameters, difficulty with mobilization - is likely to be developing a complication and this should prompt the early start of the diagnostic-therapeutic pathway to rule out or treat eventual complications. On the contrary, a patient who clinically recovers quickly and completely, whose bowel works normally (no need to wait a full bowel motion, but just the first flatus), his vital signs are normal and pain is easily controllable can be discharged very early (even on the same day if the safety network is in place) with very low risk of complications and readmission.

However, we would expect that a number of patients would develop complications after their discharge and might need to be readmitted. This does not represent a failure of the ERAS pathway, as the outcome of complicated and readmitted patients is not different from the outcome of complicated in-patients who did 
not need readmission ${ }^{[16]}$.

ERAS is by its own nature a multidisciplinary approach, where the paradigm of surgical treatment has been shifted from the surgeon to the team, consisting of surgeons, anaesthetists, physiotherapists, dieticians, physician assistants and specialist nurses. Adherence to the protocols is crucial to get the positive effects of ERAS in terms of better recovery, fewer complications, reduced LOS, reduction of costs and increased patient satisfaction ${ }^{[17]}$.

Although ERAS principles are gradually spreading to every surgical specialty, due to local or national arrangements, the initial and still greatest interest is in colorectal surgery ${ }^{[12]}$. Within this specific field, a further division has been done between colonic $^{[6]}$ and rectal ${ }^{[18]}$ surgery. Although this differentiation sounds to us a bit forced - as any excessive subspecialisation - we cannot deny that some issues specific to rectal surgery still exist and will be highlighted in the present review. With respect to colonic surgery, extraperitoneal rectal surgery is often associated with higher risk of complications due to (1) critical blood supply to the rectal stump and the proximal colonic stump in anterior resection due to anatomical reasons; (2) more difficult surgical technique and longer operation time; (3) previous pelvic radiotherapy; and (4) more frequent need for covering stoma. For these reasons, postoperative length of stay is usually longer after rectal resection with respect to colonic resection.

The ER protocols have 3 fundamental steps: (1) preoperative; (2) intraoperative; and (3) postoperative [Figure 1]. This artificial division does not reflect into the reality, as the three components overlap and interlace very often and one influences the others.

\section{PREOPERATIVE STAGE}

The preoperative phase of the ERAS protocols is crucial for the success of the whole treatment. For this reason, the full potential of ERAS can be obtained only in elective patients, as those needing an emergency operation may miss this important step. To try and extend the benefits of ER also to patients admitted as emergencies, one of the Authors endorsed a staged approach in patients with acutely complicated colorectal cancer, with an initial damage control procedure followed by an elective resection after the patient has been stabilized and fully investigated and prepared ${ }^{[19]}$.

However, quite recently, emergency operations have been included into ER protocols, as it was felt that emergency patients can benefit from some of the improvements yielded by ERAS ${ }^{[20]}$.

\section{Preoperative counseling}

One of the great advantages of ERAS is that the patient has been brought back to the centre of the whole experience and has been empowered to take care of him/herself and their recovery. The first meetings with the patient before their surgery are paramount to ensure the correct application of the ERAS protocol.

During the first encounters, after discussing diagnosis and treatment, the patient is offered to be included into the ERAS programme. Actually, the advantages of ERAS versus the traditional perioperative protocols have already been extensively demonstrated, so in our opinion there is no need to consider ERAS as a "special" measure and the ERAS principles must be considered at the basis of the standard and routine management of surgical patients. Placing special attention on ERAS - with specific paperwork - makes it appear as if it is still a sort of "experimental" treatment needing a "special" consent - which is clearly not the case.

It is true, however, that patients must be accurately informed before the operation, which is best practice anyway, of the kind of management they can expect before, during and after surgery. All the elements must be discussed, including bowel and systemic preparation, DVT and antibiotic prophylaxis, surgical 


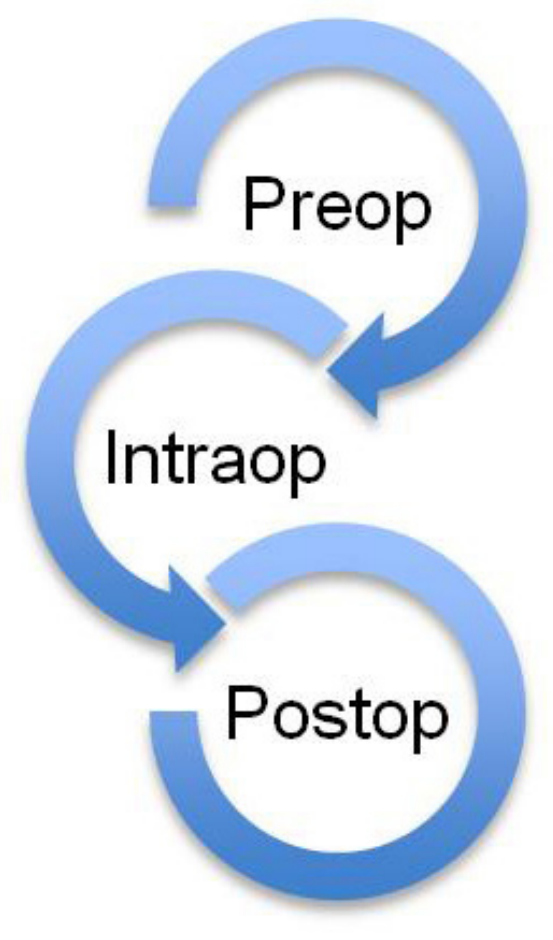

\author{
Preoperative counseling/stoma education \\ Optimization - pre-habilitation \\ Preoperative nutrition \\ Selective bowel preparation \\ No fluid overload \\ Antibiotic and DVT prophylaxis \\ Short-acting anaesthetic agents \\ Minimal use of opioids \\ Prevention of hypothermia \\ Mini-invasive technique \\ Selective use of drains, $<24 \mathrm{~h}$ \\ No nasogastric tube \\ Bladder catheter $<24 \mathrm{~h}$ \\ Multimodal postoperative analgesia \\ Ideally no morphine $>24 \mathrm{~h}$ \\ PONV prevention \\ Prevention of postoperative ileus \\ Early feeding \\ Early mobilization \\ Quality control/audit
}

Figure 1. ERAS flowchart

technique, postoperative diet, mobilization/rehabilitation and pain control. In particular, the discharge must be prepared well in advance, in the preoperative period, and all the systems must be put in place for a safe return home. Involvement of the patient and their family (not only providing information) is crucial. A recent non-blinded randomized controlled trial from Norway clearly showed the value of extensive counseling within an ERAS programme in colorectal surgery. Patients who received repeated information preoperatively showed a more efficient engagement in some elements of the ERAS programme such as early feeding and early mobilization after surgery, thus reducing their LOS by 2 days with respect to the patients who received only standard information ${ }^{[21]}$.

In our practice, the patient has at least 3 important meetings before surgery: (1) with the consultant surgeon, who communicates the diagnosis, offers and discusses the treatment and introduces ERAS; (2) with the anaesthetist, who provides preoperative optimization of the patient, discusses the pre, intra and postoperative anaesthesiological management and explains the ERAS in more detail; and (3) with the colorectal nurse who is responsible for a holistic approach and oversees some particular elements of the ERAS protocol. Sometimes the colorectal specialist nurse can be in charge of the ERAS protocol as well as the preoperative stoma teaching and site marking. In fact, the creation of a stoma is itself a contributory factor of prolonged LOS, therefore with the ERAS protocol we should aim at reducing the impact of stomas on the postoperative recovery. Stoma education is more effective if initiated in the preoperative period ${ }^{[22]}$. It is mandatory that that criteria and pathway for discharge are discussed with the patient at this stage ${ }^{[23]}$.

\title{
Optimization
}

During the preoperative meetings, all the comorbidities are fully investigated and the patient is optimized for surgery, including quitting smoking and alcohol, rebalancing their sugar levels and addressing their nutritional needs. Clearly, the optimization process needs time, and the ERAS Society guidelines suggest that 
preoperative counseling must take place 4 weeks before surgery ${ }^{[18]}$.

Despite some less than enthusiastic report ${ }^{[24]}$, pre-habilitation is becoming a key factor in increasing the functional capacity of the patient before an operation ${ }^{[25,26]}$. It has been defined as a multimodal protocol involving not only nutritional assessment and support, but also physical exercise. Typically, patients considered for prehabilitation are recruited in a 3 or 4-week supervised physical training associated with unsupervised home exercises. This schedule has been demonstrated to be beneficial to improve the cardiopulmonary exercise test scores ${ }^{[27,28]}$ and to significantly reduce LOS and length of recovery ${ }^{[28,29]}$. The effect of prehabilitation as opposed to standard rehabilitation is more evident in sedentary people ${ }^{[30]}$.

It is clear that this protocol is time-consuming and may delay curative surgery for cancer. In particular in the countries, such as the UK, where cancer targets exist and require the operation to be performed as soon as possible after the diagnosis, prehabilitation has not been diffusely accepted yet due to the fears that a prolonged preoperative interval would impair survival ${ }^{[31]}$. However, a recent prospective study from UK has demonstrated that time from diagnosis to surgery - either 4 , 8 or 12 weeks - does not impact on survival, so the regulatory pathways can safely be changed to accommodate prehabilitation ${ }^{[32]}$. A few prospective trials are still ongoing and are targeted at assessing the value of physical prehabilitation in patients scheduled for colorectal surgery ${ }^{[28,33-35]}$. We are looking forward to evaluating their results. However, it should be universally clear by now that prehabilitation should be included in every ERAS protocol, possibly adding also anxiety-reduction techniques which may benefit both the psychological and physical wellbeing of patients undergoing major rectal surgery ${ }^{[36]}$.

\section{Preoperative nutrition}

One of the new concepts highlighted by ERAS is that a correct preoperative nutrition can improve postoperative recovery by reducing the effects of surgical stress. As malnutrition is a negative prognostic factor for LOS and complications ${ }^{[37]}$, nutritional assessment and support have been included in the prehabilitation programme.

It has been demonstrated that the traditional long fasting before the operation has no advantages in terms of possible risk of inhalation ${ }^{[38]}$ and, on the contrary, can be detrimental due to the risk of increasing the physiologically stress-related insulin-resistance ${ }^{[39]}$. Randomized controlled trials demonstrated that the administration of a specific sugary fizzy drink before the operation could reduce postoperative insulin-resistance, other than hunger, thirst and anxiety ${ }^{[23]}$. The preoperative sugar load would improve the metabolism of proteins and nitrogen and reduces the detrimental catabolic effects of surgical stress on the muscular mass and the healing process $^{[40,41]}$, thus reducing the risk of complications and expediting the recovery.

Recent recommendation of the American Society of Anesthesiologists is that patients should have free access to fluids up to $2 \mathrm{~h}$ before surgery and to solid food up to $6 \mathrm{~h}$ before surgery, thus resembling a "normal" nutritional pattern ${ }^{[42]}$. Particular attention has been suggested - at least initially - to diabetic patients, where sugary drinks can potentially derange their glycaemia. Moreover, patients with complicated type 2 diabetes are known to have an increased risk of delayed gastric emptying, so - at least theoretically - the ERAS recommendations must be applied with special care.

Immuno-nutrition has been recently introduced as a way to reduce complications and improve recovery. The SONVI Study compared an immune-enhancing pre and postoperative nutrition with the traditional hyper caloric hypernitrogenous supplement within an ERAS programme and found that the number of infective complications was significantly reduced in the treated group ${ }^{[43]}$. Immuno-nutrition will be further discussed. 
Nutritional supplements are mandatory in malnourished patients and preoperative enteral or parenteral feeding should be considered in patients who are not meeting the nutritional requirements.

\section{Bowel preparation}

Mechanical bowel preparation (MBP) has always been one of the cornerstones of colorectal surgery and lower gastrointestinal endoscopy, but it may be associated with dehydration and electrolyte imbalance, in particular in high risk patients (advanced age, renal impairment, liver failure). A Cochrane systematic review published in 2005 indicated that MBP does not prevent anastomotic complications and therefore should be avoided ${ }^{[6,44]}$. Although it was clear that this review was poorly significant due to high heterogeneity, it was suggested that no patient scheduled for colorectal resectional surgery should have his/her bowel prepared. A RCT trial published in 2010 and a new Cochrane review published in 2011 found that, although bowel preparation would make no difference in colonic surgery, it may be beneficial - and hence used selectively - in patients undergoing rectal surgery ${ }^{[45,46]}$. More recent studies seem to give contrasting results.

Two recent meta-analyses on this subject, one conducted in the USA and the other in Europe, propose a totally different approach. Yost et al ${ }^{[47]}$ from Massachusetts found that MBP associated with oral antibiotics can reduce the risk of anastomotic leak, wound infection and postoperative sepsis, and facilitate the manipulation of the bowel in laparoscopic surgery. On the contrary, the systematic review by Leenen et al ${ }^{[48]}$ from the Netherlands arrives to the conclusion that MBP does not decrease the incidence of leak, but admits that the study is flawed by the small size of the sample and the heterogeneity of the studies reviewed. Already in 2015 a retrospective study on prospectively collected outcome data from the National Surgical Quality Improvement Program on 8442 patients showed that MBP almost halves the risk of anastomotic leak, ileus and surgical site infection ${ }^{[49]}$.

The different approach to this issue between the two sides of the ocean reflects in the official guidelines. The 2012 guidelines of the European ERAS Society clearly state, that MBP should be avoided in colonic and rectal surgery ${ }^{[6,18]}$. On the contrary, the 2017 ASCRS/SAGES guidelines advocate the use of MBP with oral antibiotics $^{[23]}$. This difference can be explained, at least partially, by the fact that the US review took into account more recent evidences, clearly not available 5 years before, but also by the different cultural, and probably financial attitude. Although local guidelines must be developed and validated, it is undeniable that at the moment this is still matter for debate and a definitive answer is yet to come. We feel that with the current status of evidence the ultimate choice must stay with the consultant in charge, as some surgeons may feel more confident to operate on a completely empty and deflated bowel, mostly in laparoscopy, whereas others do not see the presence of a non-prepared bowel as a limitation or increasing difficulty. The role of oral antibiotics associated with MBP is gaining popularity after the studies of Alverdy and his team in Chicago ${ }^{[50]}$ and the ERAS guidelines of the ASCRS/SAGES ${ }^{[23]}$.

\section{INTRAOPERATIVE STAGE}

\section{Intra and postoperative fluid therapy}

The traditional liberal infusion of fluids during and after a major operation, either by open or laparoscopic surgery, has been demonstrated to be one of the causes of prolonged postoperative ileus, probably due to the tissue oedema or sodium excess, and other perioperative complications, therefore is has been suggested to have a more restrictive fluid regimen ${ }^{[51,52]}$. The so called "goal-directed fluid therapy" has been considered as the first choice to correctly titrate the amount of fluids to be infused during and after a surgical operation ${ }^{[53]}$. Invasive and non-invasive monitoring systems have been used to utilize cardiac output as a guide for fluid infusion ${ }^{[1,54]}$, but currently most teams prefer to adopt a more "empiric" system, with a low volume of intraoperative infusions followed by free oral fluid in the immediate postoperative period, 
at least in elective patients with no important comorbidities. Obviously, cardiac output remains the best indicator of volaemia (and oxygen delivery), but pulse, blood pressure and urine output are generally considered good enough to guide fluid infusion in rectal surgery. The growing diffusion of laparoscopic surgery, which entails reduced evaporative fluid loss and reduced blood loss with respect to open surgery, has helped significantly to reduce the amount of volume to be infused. However, the recent RELIEF trial failed to demonstrate a higher rate of disability-free survival and pointed out an increased risk of acute renal failure in patients with restricted fluid infusion ${ }^{[5]}$. Although this interesting study is gradually contributing to switching the paradigm of restrictive fluid infusion, we can reasonably admit that at the moment the pathophysiology of fluid infusion during surgery is not yet completely understood and we may still decide case by case on empiric bases until more definitive evidence becomes available.

The vast majority of published articles do not differentiate between colonic and rectal surgery, but rectal surgery is more easily associated with dehydration or hypovolaemia due to increased duration of surgery, bowel preparation and possible blood loss in the pelvis, therefore a less restrictive fluid infusion may be advisable.

Our attitude is to reasonably limit the intraoperative infusion to maintain adequate tissue perfusion during the operation and to allow the patient to consume free or clear fluids as soon as he or she is awake and comfortable in the recovery room after surgery. Invasive monitoring of the cardiac dynamics increases the risks, delays the mobilization and raises the costs of surgery, but it may be necessary in selected cases, mostly in unprepared patients.

\section{Antibiotic prophylaxis and DVT prophylaxis}

It has been widely demonstrated that systemic antibiotic prophylaxis reduces the risk of postoperative infections ${ }^{[56]}$. Both ultra-short term prophylaxis (a single dose before surgery) and short term prophylaxis (a preoperative dose plus $24 \mathrm{~h}$ coverage after surgery) revealed effective. It is still a matter for debate what antibiotics should be used. Clearly, they must be active on aerobic and anaerobic so usually a combination of two antibiotics is needed, but the choice is usually left with local policies and protocols. The first dose should be administered within $1 \mathrm{~h}$ from skin incision. Apparently, the association of intravenous and oral antibiotic is more effective than intravenous alone ${ }^{[57]}$. Much debate has been done recently on the use of intraluminal antibiotics in association with mechanical bowel preparation (see dedicated paragraph), which would be able to reduce the risk of ileus and leak.

DVT prophylaxis has been one of the pivots of the traditional perioperative management for decades. It is well known that the correct administration of low-molecular-weight heparin and the use of thromboembolism deterrent stockings (TEDS) would decrease the risk of DVT and pulmonary embolism. Obviously, this best practice has been integrated into the ERAS protocols.

\section{Anaesthesia}

Open rectal surgery usually needs a long abdominal incision, a wide pelvic dissection and sometimes also a perineal incision with severe disruption of the pelvic floor. The laparoscopic approach reduces the trauma of the midline laparotomy, even if often a small laparotomy may still be needed, but increases the operative time and adds the further pathophysiologic trauma of a steep Trendelenburg position.

The ERAS principles pertaining to anaesthesia are: (1) short-acting agents to expedite the postoperative recovery; and (2) optimal pain control with minimal use of opioids. Intraoperative pain relief can be obtained with a blended approach with epidural analgesia (see postoperative analgesia) and short acting opioids. Remifentanyl can reduce the surgical trauma and the stress response. Muscle relaxation is essential in 
laparoscopic surgery of the pelvis - much more than in open surgery - to allow a complete distension of the abdomen without contrasting the $\mathrm{CO}_{2}$ insufflation and to facilitate the pelvic dissection ${ }^{[23]}$.

\section{Prevention of hypothermia}

It is well known that hypothermia is one of the killers of the surgical and trauma patient, also due to its negative effect on coagulation. It has been demonstrated, and is considered best practice, that prevention of hypothermia through the administration of warm fluids and the use of a thermic blanket can reduce bleeding, surgical site infection and cardiac complications.

Hypothermia can also be detrimental in terms of prolonging the admission and delaying oral feeding, but its direct relation with postoperative ileus has not been proved ${ }^{[58]}$. It has been suggested to monitor body temperature during and after surgery ${ }^{[18]}$.

\section{Surgical technique}

The ERAS being mostly a perioperative policy, the surgical technique is pretty much unchanged with respect to the traditional protocols. However, if we want to get the whole range of benefits of ERAS, it is clear that the surgical technique must be as less invasive as possible. Therefore, the higher benefits of ERAS can be obtained in patients operated by laparoscopy ${ }^{[2,5]}$. Although pelvic dissection is always traumatic, in particular when coupled with perineal dissection, the laparoscopic approach associated with ERAS would reduce postoperative ileus and overall physiologic recovery ${ }^{[18]}$. Numerous and strong evidence ${ }^{[59,60]}$ confirmed that laparoscopic surgery for rectal cancer is feasible, safe and effective and adds the benefits of the miniinvasive approach to the care of patients with rectal malignancies. The combination of laparoscopic surgery with the ERAS approach improves the already outstanding results of each of the two taken singularly; therefore we strongly believe that laparoscopic surgery must be considered one of the core items of ERAS also in pelvic benign and malignant conditions.

\section{Use of drains}

If one of the principles of the surgical technique under an ERAS protocol is to be respectful of the particular pathophysiology of the patient, it necessarily follows that all those items that can delay his or her recovery should be avoided. The presence of one or more drains in the pelvis can impair the patient's early mobilization, without reducing the incidence or the severity of anastomotic leaks ${ }^{[61,62]}$. A large retrospective study from Holland and a meta-analysis from Italy showed that in patients who had total mesorectal excision the use of drain can be beneficial ${ }^{[63,64]}$, but this finding was not confirmed by the GRECCAR 5 randomized controlled trial ${ }^{[65]}$.

\section{Nasogastric tube}

The traditional use of a nasogastric (NG) tube to prevent postoperative vomiting and gastric distension has been demonstrated to be detrimental to an expedited recovery as it may be one of the causes of respiratory complications. A Cochrane meta-analysis demonstrated that bowel motility recovers quickly without a NG tube, with no increased risk of inhalation ${ }^{[6]}$. NG tubes should be reserved to those patients who develop postoperative ileus ${ }^{[23]}$.

\section{Bladder catheter}

The use of a bladder catheter (either transurethral or suprapubic) has been part of the traditional perioperative management for decades, as it allows to monitor the urinary output and to deflate the bladder to improve the visualization of the pelvis, either by open or laparoscopic surgery. Moreover, it can be useful if the patient is not yet fully mobile. In rectal surgery, bladder catheter is standard practice due to the risk of urinary function impairment. However, there is no evident advantage in maintaining the catheter beyond 
the strict necessity. Several studies, and consequently the ERAS guidelines, suggest taking it out as soon as possible, even within the 1 st day ${ }^{[18,67]}$. The American guidelines differentiate colonic and rectal surgery and suggest removal of the bladder catheter within day 2 in infraperitoneal rectal resections. Obviously, a longer duration of bladder catheterization may be necessary in patients with increased risk of urinary retention $^{[18]}$, such as in the presence of epidural analgesia, and in those who had extensive pelvic surgery ${ }^{[23]}$.

\section{Prevention of postoperative infections}

Colorectal surgery patients are more prone to develop infections than any other surgical patients, possibly due to the high potential for bacterial seeding and/or translocation. Whereas specific infective processes such as pneumonia have a clear pathophysiology, it is still not perfectly clear why patients develop surgical site infection (SSI). The traditional view that this may be due to direct contamination during the operation, suspected on the basis that the most common causative bacteria of SSI are enteric in origin (E. coli, B. fragilis), has been disproved by the fact that cultures of the wound at the end of a surgical operation are not predictive of postoperative infection ${ }^{[68]}$. It has been proposed a "Trojan Horse" hypothesis for SSI, whereby virulent bacteria normally quiescent within the gastrointestinal tract are simply transferred to the surgical wound by neutrophils and macrophages activated by the surgical trauma ${ }^{[69]}$. The use of oral non-absorbable antibiotic would therefore be beneficial also in reducing the risk of $\operatorname{SSI}^{[23,50]}$.

A similar mechanism has been invoked for the genesis of anastomotic leak. It has been demonstrated long ago that bacteria and not technique are usually responsible for anastomotic leak ${ }^{[70]}$. Unfortunately, for some reasons the view that local ischaemia and technical failure are the main causative factor for leak prevailed, despite the clinical evidence that oral antibiotics would decrease the risk of leak ${ }^{[71]}$, until the more recent evidences $^{[49]}$ convinced the American Society of Colon and Rectal Surgeons and the Society of American Gastrointestinal and Endoscopic Surgeons to include oral antibiotics in their ERAS guidelines ${ }^{[23]}$.

The adoption of a bundle of measures to reduce SSI is a winning entry. A recent systematic review and meta-analysis showed a reduction of the risk of SSI of more than $50 \%(15.1 \% v s .7 \%)$. All the studies considered in this review had in common the core elements of the SSI-reduction bundles, namely antibiotic prophylaxis, prevention of hypothermia, hair removal, prevention of hyperglycaemia ${ }^{[72]}$. Other interventions have been suggested, such as no fluid overload, skin preparation with chlorhexidine, double gloving or change of gloves and gowns before closing the fascia, lavage of subcutaneous tissue and silver dressing ${ }^{[23]}$.

\section{POSTOPERATIVE STAGE}

\section{Postoperative analgesia}

Control of postoperative pain has always been a hot topic, as it is one of the variables that may affect postoperative recovery. The traditional use of morphine has always been quite effective, but at the expense of important side effects, such as prolonged ileus, and potential complications (respiratory, neurologic) and safety issues (drowsiness, dizziness, falls) in high risk patients. Several different analgesia regimens have been proposed, with the aim of minimizing patient discomfort while facilitating his or her recovery and minimizing side effects and complications of drugs. We agree with the American Society for Enhanced Recovery (ASER) that a completely painless surgery is a non-achievable goal ${ }^{[73]}$, so we should aim at the best possible analgesia that does not impair the patient's physical recovery. Every analgesic regimen must also take into consideration the kind of surgical approach, as the requirements of pain control may vary according to the size and shape of the abdominal incision and the subsequent surgical trauma.

Opioids have been extensively used in the past to control postoperative pain, but at the expense of heavy 
side effects such as constipation, ileus, nausea, urinary retention, over sedation, delirium and delayed discharge. Clearly, this does not fit with the ERAS principles. The further demonstration, that non-opioid analgesia may be associated with less pain, reduced risk of cancer recurrence and longer survival ${ }^{[7]}$ has convinced the medical community that non-opioids regimens should be considered. The European guidelines suggest using opioids only as rescue analgesia, in particular in laparoscopic surgery. The ASER guidelines suggest a multimodal approach with NSAIDs, paracetamol and/or gabapentin, eventually associated with spinal/epidural analgesia and/or local anaesthetic infusion within the wound. Eventual adjuncts can be steroids, ketamine and tramadol ${ }^{[73]}$.

It has been demonstrated that the neuro-axial block is able to reduce the surgical stress by interrupting the transmission of the nociceptive stimulus to the brainstem, thus reducing the risk of postoperative ileus ${ }^{[2,75]}$. Epidural analgesia is also effective for the pain control in the postoperative period, even if many Authors prefer to limit its use to laparotomic surgery where its benefits are definitely clearer than the risks. Epidural anaesthesia (EA) is associated with very good pain control after colorectal surgery, both laparoscopic and open, but the prolonged block of the sympathetic system, with consequent hypotension and need for continuous fluid infusion, is a major concern. Although the benefits of EA are well known ${ }^{[76]}$, the use of single-injection intrathecal (spinal) analgesia (IA) with opioid plus or minus local anaesthesia is gaining favor $^{[77]}$. The main advantage of IA with respect to epidural is its easiness, as it does not require any further equipment or specific surveillance. The perceived drawback is its potential risk of complications such as respiratory depression. This has been widely contradicted by scientific evidences ${ }^{[77]}$. On the contrary, IA is safe and effective, as it is able to control postoperative pain while allowing earlier functional recovery and shorter $\operatorname{LOS}^{[78]}$. IA is usually performed to provide additional pain control in association with paracetamol and NSAIDs.

Peripheral analgesia can also be extremely effective. Transversus abdominis plane infiltration, wound and peritoneal infiltration and rectus sheath block in association with systemic analgesia have all been demonstrated to be able to reduce the use of opioid with respect to systemic analgesia only ${ }^{[73]}$.

All non-opioid oral analgesic agents have been widely used and have been found to be able to reduce the use of opioids. Their routine use is part of any ERAS programme. Intravenous administration of paracetamol can achieve higher plasma concentrations with respect to the oral administration, but there is no evidence of a clear superiority of intravenous versus oral administration in the clinical settings ${ }^{[79]}$. The use of NSAIDs has been recommended on the basis of good efficacy and insufficient evidence of complications $^{[80]}$. However, recent evidence suggest caution with the use of non-selective NSAIDs like diclofenac, due to increased risk of anatomotic leak ${ }^{[81]}$. Gabapentin and pregabalin can also be used to reduce the requirement of opioids in the postoperative period ${ }^{[82]}$, and as such their use is recommended by the ASER ${ }^{[73]}$, but only at high doses. Tramadol ${ }^{[83]}$, ketamine $^{[84]}$, magnesium ${ }^{[85]}$ and steroids ${ }^{[86]}$ have all been used for postoperative pain control, with some efficacy, but their use has not gained wide acceptance.

\section{Prevention of postoperative nausea and vomiting}

Postoperative nausea and vomiting (PONV) are common side effects of surgery and anaesthesia and can affect early feeding and reduce patient satisfaction. Recent guidelines suggest a multimodal and quite liberal approach to PONV treatment ${ }^{[23]}$. Avoidance of opiates and administration of oxygen can help reducing PONV. Goll et al. ${ }^{[87]}$ demonstrated that $2 \mathrm{~h}$ of $80 \%$ oxygen are more effective in reducing PONV than ondansetron. However, postoperative ondansetron following preoperative administration of dexamethasone is an effective combination ${ }^{[23]}$. It is well known that intravenous anaesthesia with propofol is superior to inhalation anaesthesia ${ }^{[88]}$, but the role of gabapentin is not yet fully defined ${ }^{[23]}$. 


\section{Prevention of postoperative ileus}

Postoperative ileus still remains a major problem after colorectal surgery, and in particular after extensive rectal operations, with a prevalence rate as high as $28 \%{ }^{[89]}$. Its global impact on LOS overlaps the impact of anastomotic leak ${ }^{[90]}$. Its etiology is multifactorial and usually is not related to a substandard surgical technique. Smoking, major laparotomy, ASA classification and duration of surgery were considered independent causative factors ${ }^{[91,92]}$. Molecular level evidence has recently demonstrated the role of intestinal microbioma in the regulation of bowel motility ${ }^{[93]}$, possibly through its action on bowel macrophages, whose activation leads to generalized intestinal nervous plexa dysfunction and paralytic ileus ${ }^{[94]}$. This can be taken as a confirmation of the positive role of oral non-absorbable antibiotics in the preparation of the bowel for colorectal surgery ${ }^{[49]}$, even if the exact mechanism of action and the bacteria species involved in the process are not yet fully understood ${ }^{[50]}$.

Compliance with ERAS principles is a protective factor against postoperative ileus ${ }^{[95]}$. According to ERAS principles, early recovery of bowel peristalsis is based on four cornerstones: (1) mini-invasive technique; (2) restrictive fluid infusion; (3) avoidance of opiates; and (4) early re-feeding.

Multiple RCTs and meta-analyses have demonstrated that early feeding stimulates recovery of bowel function $^{[23,96]}$; this is particularly true in younger patients operated on by subspecialist colorectal surgeons using laparoscopic techniques ${ }^{[97,98]}$.

It is well known that opioids may impair the gastrointestinal motility through a direct action on the mureceptors of the bowel, causing ileus and constipation. Therefore, the use of peripheral opioid antagonists may reduce the risk of postoperative ileus ${ }^{[97]}$. Although this has been incorporated into the American ERAS guidelines $^{[23]}$, some Authors suggest prudence as definitive evidence is not yet available ${ }^{[98]}$. A recent phase 2 study from Scotland confirmed that oxycodone and naloxone reduces time to first bowel motion when compared to oxycodone only within an ERAS protocol in laparoscopic colorectal surgery, but the authors admit that a proper RCT is still needed ${ }^{[99]}$.

It has been proposed that chewing gum may stimulate bowel motility - via vagal stimulation or gastrointestinal hormones secretion - with an earlier return of intestinal function and reduced LOS $^{[100]}$, but unfortunately the vast majority of colorectal studies are small sized and of poor quality ${ }^{[101,102]}$. A large RCT on 402 patients clearly demonstrated that, although there is no detriment, there is no beneficial effect either ${ }^{[103]}$. Although chewing gum has been added to the ERAS Society guidelines ${ }^{[18]}$, this matter remains unclear.

The use of oral laxatives such as magnesium oxide and disodium phosphate can reduce the time to first bowel motion ${ }^{[104]}$, but it has not been fully evaluated yet and it is considered a weak recommendation ${ }^{[18]}$.

\section{Postoperative nutrition}

Early studies confirmed that early oral feeding after a colorectal operation does not increase the risk of complications and, on the contrary, can be beneficial in reducing the post-surgical catabolism, improving the immune function, reducing the systemic inflammatory response and reducing bacterial translocation. The ERAS protocols suggest early re-feeding with liberal fluid intake by mouth immediately after surgery, provided that PONV is well controlled, and early return to a normal diet ${ }^{[18]}$. In cases where oral feeding is not deemed to be sufficient to get a correct caloric intake, it may be necessary to add oral proteic supplements $^{[18]}$.

Even if the application of simple rules can yield great results, the implementation of a specific gastrointesti- 
nal rehabilitation programme resulted in further reduced morbidity and $\operatorname{LOS}^{[105]}$.

Immunonutrition is associated with reduced rate of complications and reduced length of stay ${ }^{[106,107]}$. In fact, it has been demonstrated that the administration of nutritional supplements containing mainly glutamine, arginine, omega-3 fatty acid and ribonucleic acid is able to reduce the inflammation and improve the immune response. The mechanism of action of immunonutrition has not been fully clarified yet. Apparently, its main effect is to reduce bacterial translocation by maintaining the integrity of the intestinal mucosa and reducing its permeability, thus reducing the risk of infectious complications ${ }^{[107]}$.

The main barrier to a correct postoperative nutrition is patient information ${ }^{[108]}$, so once again it is important to emphasize the need for patient engagement also in nutritional care at the preoperative encounters $^{[109]}$.

\section{Early mobilization}

Early postoperative mobilization has beneficial effects on the whole postoperative recovery. In fact, it reduces catabolism and bowel recovery and improves ventilator function and bronchial clearance (reducing the risk of pneumonia and atelectasis), reduces the risk of DVT and prevents muscle loss and insulinresistance. Ultimately, it reduces postoperative LOS, encourages independence and reduces discomfort ${ }^{[18]}$. Several different modalities and targets have been proposed ${ }^{[23]}$, but no specific protocol has been identified. A frequent approach is to allow liberal mobilization as soon as possible after surgery ${ }^{[23]}$, but in high risk patients, strong support by specialized staff may be needed.

\section{Quality control}

Auditing outcome data is the only way to improve the service we are offering. Once an ERAS programme has been started, it is likely that this will become part of the "routine" management of surgical patients. Nonetheless, there is good recent evidence that habituation can lead to over-confidence and the application of some of the ERAS elements can reduce with time ${ }^{[110,111]}$. The exact quality indicators of ERAS protocols are yet to be defined, but it is obvious that LOS is not necessarily the main endpoint. Fit-for-discharge as a parameter can be more reliable than $\operatorname{LOS}^{[112]}$. The ERAS Society recommends also considering morbidity and mortality, need for transfusions, duration of surgery, readmission rate and total cost. We would also add reoperation rate and patient satisfaction as key factors for quality control. Moreover, it must be emphasized that the ultimate aim of rectal resection is to cure a malignant condition; therefore we believe that oncologic outcomes, such as rate of radical resections and rate of adequate lymphadenectomies must also be taken as quality parameters.

Another parameter that must be recorded and audited is the compliance with the elements of ERAS. Engagement of the whole multidisciplinary department is paramount and adherence to the process is crucial to obtain the advantages of ERAS ${ }^{[17,113]}$.

To the best of our knowledge, adherence to ERAS protocols has never been analysed in relation to the size of the department, however we feel that in a small department it should be relatively easy to implement the necessary changes leading to the full potential of ERAS principles, with respect to a big-size department where engagement of all the relevant people can be tricky and sometimes frustratingly impossible. One of the Authors' past experience with implementing ERAS in a rural hospital has shown that evolutionary changes aiming at improving postoperative recovery in colorectal surgery can be implemented relatively quickly, and the learning curve of the whole team is quite short ${ }^{[5]}$. In more sizeable departments, the introduction of the ERAS protocol should be more gradual, focusing initially on prevention of hypothermia, early postoperative feeding, early removal of the bladder catheter and no nasogastric tube ${ }^{[14]}$. Actually, the Perioperative Italian Society who released this statement in a very recent publication added no bowel 
preparation to the "core" items listed above, but on this subject we favour the American guidelines, suggesting selective bowel preparation. Although a gradual approach can be acceptable, we again agree with the American guidelines that the aim must be a complete and standardised protocol implementation ${ }^{[23]}$.

Probably never, have such minimal changes in surgical organisation and mindset yielded such a huge return as with ERAS in colorectal surgery ${ }^{[4]}$.

\section{CONCLUSION}

ERAS protocols are largely improving the experience of patients undergoing colorectal surgery but they also represent a huge step forward in team working, hospital dynamics and trust finances, as they reduce LOS, total hospital costs and pharmacy costs, with a contemporary reduction of complications and readmission rates ${ }^{[115]}$. In a world where new scientific evidences are published on daily basis it is normal that ERAS principles are still quite fluid, but this is not necessarily a bad thing. Some of the ERAS elements are particularly likely to change in the near future, such as the attitude towards MBP and oral antibiotic, prehabilitation and immunonutrition. We can expect that with increased experience and awareness, LOS will reduce with time and more and more patients will be operated as day cases in the future.

Despite the various attempts at standardisation, ERAS statements remain general principles whose dynamic application must be adapted to the local situation and to the team preferences and must show an ample degree of flexibility. For this reason, every team applying ERAS standards must keep in mind the need for regular audits, quality checks and improvement.

\section{DECLARATIONS}

\section{Author's contributions}

Literature selection and review, article drafting, and approved the final version: Tebala GD, Gordon-Dixon A, Imtiaz M, Shrestha A, Toeima M

\section{Availability of data and materials}

Not applicable.

\section{Financial support and sponsorship}

None.

\section{Conflicts of interest}

The authors declare that there are no conflicts of interest.

\section{Ethical approval and consent to participate}

Not applicable.

\section{Consent for publication}

Not applicable.

\section{Copyright}

(c) The Author(s) 2018.

\section{REFERENCES}

1. Kehlet H, Wilmore DW. Multimodal strategies to improve surgical outcome. Am J Surg 2002;183:630-41.

2. Kehlet H, Wilmore DW. Evidence-based surgical care and the evolution of fast-track surgery. Ann Surg 2008;248:189-98. 
3. Khreiss W, Huebner M, Cima RR, Dozois ER, Chua HK, Pemberton JH, Harmsen WS, Larson DW. Improving conventional recovery with enhanced recovery in minimally invasive surgery for rectal cancer. Dis Colon Rectum 2014;57:557-63.

4. Thiele RH, Rea KM, Turrentine FE, Friel CM, Hassinger TE, McMurry TL, Goudreau BJ, Umapathi BA, Kron IL, Sawyer RG, Hedrick TL. Standardization of care: impact of an enhanced recovery protocol on length of stay, complications, and direct costs after colorectal surgery. J Am Coll Surg 2015;220:430-43.

5. Tebala GD, Keane S, Osman A, Ip M, Khan AQ, Perrone L. Early discharge after colorectal resection: the positive impact of an enhanced recovery program on a rural colorectal surgery service. Surg Laparosc Endosc Percutan Tech 2016;26:e137-44.

6. Gustafsson UO, Scott MJ, Schwenk W, Demartines N, Roulin D, Francis N, McNaught CE, MacFie J, Liberman AS, Soop M, Hill A, Kennedy RH, Lobo DN, Fearon K, Ljungqvist O; Enhanced Recovery After Surgery Society. Guidelines for perioperative care in elective colonic surgery: enhanced recovery after surgery (ERAS $($ ) society recommendations. Clin Nutr 2012;31:783-800.

7. Greco M, Capretti G, Beretta L, Gemma M, Pecorelli N, Braga M. Enhanced recovery programme in colorectal surgery: a meta-analysis of randomised controlled trials. World J Surg 2014;38:1531-41.

8. Muller S, Zalunardo MP, Hubner M, Clavien PA, Demartines N; Zurich Fast Track Study Group. A fast track program reduces complications and length of hospital stay after open colonic surgery. Gastroenterology 2009;136;842-7.

9. Serclová Z, Dytrych P, Marvan J, Nová K, Hankeová Z, Ryska O, Slégrová Z, Buresová L, Trávníková L, Antos F. Fast-Track in open intestinal surgery: prospective randomized study(clinical trials gov identifier no. NCT00123456). Clin Nutr 2009;28:618-24.

10. Teeuwen PH, Bleichrodt RP, Strik C, Groenewoud JJ, Brinkert W, van Laarhoven CJ, van Goor H, Bremers AJ. Enhanced recovery after surgery (ERAS) versus conventional postoperative care in colorectal surgery. J Gastrointest Surg 2010;14:88-95.

11. Gustafsson UO, Hausel J, Thorell A, Ljungqvist O, Soop M, Nygren J; Enhanced Recovery After Surgery Study Group. Adherence to the enhanced recovery after surgery protocol and outcomes after colorectal cancer surgery. Arch Surg 2011;146:571-7.

12. Grocott MP, Martin DS, Mythen MG. Enhanced recovery pathways as a way to reduce surgical morbidity. Curr Opin Crit Care 2012;18:385-92.

13. Forsmo HM, Pfeffer F, Rasdal A, Østgaard G, Mohn AC, Körner H, Erichsen C. Compliance with enhanced recovery after surgery criteria and preoperative and postoperative counselling reduces length of hospital stay in colorectal surgery: results of a randomized controlled trial. Colorectal Dis 2016;18:603-11.

14. Simpson JC, Moonesinghe SR, Grocott MP, Kuper M, McMeeking A, Oliver CM, Galsworthy MJ, Mythen MG; National Enhanced Recovery Partnership Advisory Board. Enhanced recovery from surgery in the UK: an audit of the enhanced recovey partnership programme 2009-2012. Br J Anaesth 2015;115:560-8.

15. Khan MA, Pandev S. Clinical outcomes of the very elderly undergoing enhanced recovery programmes in elective colorectal surgery. Ann R Coll Surg Engl 2016;98:29-33.

16. Kiran RP, Delaney CP, Senagore AJ, Steel M, Garafalo T, Fazio VW. Outcomes and prediction of hospital readmission after intestinal surgery. J Am Coll Surg 2004;198:877-83.

17. Portinari M, Ascanelli S, Targa S, Dos Santos Valgode EM, Bonvento B, Vagnoni E, Camerani S, Verri M, Volta CA, Feo CV. Impact of a colorectal enhanced recovery program implementation on clinical outcomes and institutional costs: a prospective cohort study with retrospective control. Int J Surg 2018;53:206-13.

18. Nygren J, Thacker J, Carli F, Fearon KC, Norderval S, Lobo DN, Ljungqvist O, Soop M, Ramirez J; Enhanced Recovery After Surgery Society. Guidelines for perioperative care in elective rectal/pelvic surgery: enhanced recovery after surgery (ERAS $\left.{ }^{\circ}\right)$ society recommendations. Clin Nutr 2012;31:801-16.

19. Tebala GD, Natili A, Gallucci A, Brachini G, Khan AQ, Tebala D, Mingoli A. Emergency treatment of complicated colorectal cancer. Cancer Manag Res 2018;10:827-38.

20. Shida D, Tagawa K, Inada K, Nasu K, Seyama Y, Maeshiro T, Miyamoto S, Inoue S, Umekita N. Modified enhanced recovery after surgery (ERAS) protocols for patients with obstructive colorectal cancer. BMC Surg 2017;17:18.

21. Forsmo HM, Erichsen C, Rasdal A, Tvinnereim JM, Körner H, Pfeffer F. Randomized controlled trial of extended perioperative counseling in enhanced recovery after colorectal surgery. Dis Colon Rectum 2018;61:724-32.

22. Chaudhri S, Brown L, Hassan I, Horgan AF. Preoperative intensive, community-based vs. traditional stoma education: a randomized, controlled trial. Dis Colon Rectum 2005;48:504-9.

23. Carmichael JC, Keller DS, Baldini G, Bordeianou L, Weiss E, Lee L, Boutros M, McClane J, Feldman LS, Steele SR. Clinical practice guidelines for enhanced recovery after colon and rectal surgery from the american society of colon and rectal surgeons and Ssociety of american gastrointestinal and endoscopic surgeons. Dis Colon Rectum 2017;60:761-84.

24. Singh F, Newton RU, Baker MK, Spry NA, Taaffe DR, Galvão DA. Feasibility and efficacy of presurgical exercise in survivors of rectal cancer scheduled to receive curative resection. Clin Colorectal Cancer 2017;16:358-65.

25. Minnella EM, Bousquet-Dion G, Awasthi R, Scheede-Bergdahl C, Carli F. Multimodal prehabilitation improves functional capacity before and after colorectal surgery for cancer: a five-year research experience. Acta Oncol 2017;56:295-300.

26. Carli F, Charlebois P, Stein B, Feldman L, Zavorsky G, Kim DJ, Scott S, Mayo NE. Randomized clinical trial of prehabilitation in colorectal surgery. Br J Surg 2010;97:1187-97.

27. Dunne DF, Jack S, Jones RP, Jones L, Lythgoe DT, Malik HZ, Poston GJ, Palmer DH, Fenwick SW. Randomized clinical trial of prehabilitation before planned liver resection. Br J Surg 2016;103:504-12.

28. Berkel AEM, Bongers BC, van Kamp MS, Kotte H, Weltevreden P, de Jongh FHC, Eijsvogel MMM, Wymenga ANM, BigirwamunguBargeman M, van der Palen J, van Det MJ, van Meeteren NLU, Klaase JM. The effect of prehabilitation versus usual care to reduce postoperative complications in high-risk patients with colorectal cancer or dysplasia scheduled for elective colorectal resection: study protocol of a randomized controlled trial. BMC Gastroenterol 2018;18:29.

29. Gillis C, Buhler K, Bresee L, Carli F, Gramlich L, Culos-Reed N, Sajobi TT, Fenton TR. Effects of nutritional prehabilitation, with and without exercise, on outcomes of patients who undergo colorectal surgery: a systematic review and meta-analysis. Gastroenterology 
2018;155:391-410.

30. Bousquet-Dion G, Awasthi R, Loiselle SE, Minnella EM, Agnihotram RV, Bergdahl A, Carli F, Scheede-Bergdahl C. Evaluation of supervised multimodal prehabilitation programme in cancer patients undergoing colorectal resection: a randomized control trial. Acta Oncol 2018;57:849-59.

31. Leong KJ, Chapman MAS. Current data about the benefit of prehabilitation for colorectal cancer patients undergoing surgery are not sufficient to alter the NHS cancer waiting targets. Colorect Dis 2017;19:522-4.

32. Curtis NJ, West MA, Salib E, Ockrim J, Allison AS, Dalton R, Francis NK. Time from colorectal cancer diagnosis to laparoscopic curative surgery - is there a safe window for prehabilitation? Int J Colorectal Dis 2018;33:979-83.

33. Merki-Künzli C, Kerstan-Huber M, Switalla D, Gisi D, Raptis DA, Greco N, Mungo G, Wirz M, Gloor S, Misirlic M, Breitenstein S, Tschuor C. Assessing the value of prehabilitation in patients undergoing colorectal surgery according to the enhanced recovery after surgery (ERAS) pathway for the improvement of postoperative outcomes: protocol for a randomised controlled trial. JMIR Res Protoc 2017;6:e199.

34. Wong SG, Maida E, Harvey D, Wagner N, Sonnadara R, Amin N. Evaluation of a physiatrist-directed prehabilitation intervention in frail patients with colorectal cancer: a randomized pilot study protocol. BMJ Open 2017;7:e015565.

35. Onerup A, Angenete E, Bock D, Börjesson M, Fagevik Olsén M, Grybäck Gillheimer E, Skullman S, Thörn SE, Haglind E, Nilsson H. The effect of pre- and post-operative physical activity on recovery after colorectal cancer surgery (PHYSSURG-C): study protocol for a randomized controlled trial. Trials 2017;18:212

36. Minnella EM, Carli F. Prehabilitation and functional recovery for colorectal cancer patients. Eur J Surg Oncol 2018;44:919-26.

37. Yeung SE, Hilkewich L, Gillis C, Heine JA, Fenton TR. Protein intakes are associated with reduced length of stay: a comparison between enhanced recovery after surgery (ERAS) and conventional care after elective colorectal surgery. Am J Clin Nutr 2017;106:4451.

38. Ljungqvist O, Søreide E. Perioperative fasting. Br J Surg 2003;90:400-6.

39. Black PR, Brooks DC, Bessey PQ, Wolfe RR, Wilmore DW. Mechanisms of insulin resistance following injury. Ann Surg 1982;196:420-35.

40. Crowe PJ, Dennison A, Royle GT. The effect of pre-operative glucose loading on postoperative nitrogen metabolism. Br J Surg 1984;71:635-7.

41. Svanfeldt M, Thorell A, Hausel J, Soop M, Rooyackers O, Nygren J, Ljungqvist O. Randomized clinical trial of the effect of preoperative oral carbohydrate treatment on postoperative whole-body protein and glucose kinetics. Br J Surg 2007;94:1342-50.

42. Practice guidelines for preoperative fasting and the use of pharmacologic agents to reduce the risk of pulmonary aspiration: application to healthy patients undergoing elective procedures:an updated report by the american society of anesthesiologists task force on preoperative fasting and the use of pharmacologic agents to reduce the risk of pulmonary aspiration. Anesthesiology 2017;126:376-93.

43. Moya P, Soriano-Irigaray L, Ramirez JM, Garcea A, Blasco O, Bianco FJ, Brugiotti C, Miranda E, Arroyo A. Preoperative standard oral nutrition supplements versus immunonutrition in patients undergoing colorectal resection in an enhanced recovery (ERAS) protocol: a multicenter randomized clinical trial (SONVI study). Medicine (Baltimore) 2016;95:e3704.

44. Guenaga KF, Matos D, Castro AA, Atallah AN, Wille-Jørgensen P. Mechanical bowel preparation for elective colorectal surgery. Cochrane Databasae Syst Rev 2005; doi: 10.1002/14651858.CD001544.pub2.

45. Bretagnol F, Panis Y, Rullier E, Rouanet P, Berdah S, Dousset B, Portier G, Benoist S, Chipponi J, Vicaut E; French Research Group of Rectal Cancer Surgery (GRECCAR). Rectal cancer surgery with or without bowel preparation: the French GRECCAR III multicenter single-blinded randomized trial. Ann Surg 2010;252:863-8.

46. Güenaga KF, Matos D, Wille-Jørgensen P. Mechanical bowel preparation for elective colorectal surgery. Cochrane Database Syst Rev 2011; doi: 10.1002/14651858.CD001544.pub4.

47. Yost MT, Jolissaint JS, Fields AC, Whang EE. Mechanical and oral antibiotic bowel preparation in the era of mini-invasive surgery and enhanced recovery. J Laparoendosc Adv Surg Tech A 2018;28:491-5.

48. Leenen JPL, Hentzen JEKR, Ockhuijsen HDL. Effectiveness of mechanical bowel preparation versus no preparation on anastomotic leakage in colorectal surgery: a systematic review and meta-analysis. Updates Surg 2018; doi: 10.1007/s13304-018-0526-4.

49. Kiran RP, Murray AC, Chiuzan C, Estrada D, Forde K. Combined preoperative mechanical bowel preparation with oral antibiotics significantly reduces surgical site infection, anastomotic leak, and ileus after colorectal surgery. Ann Surg 2015;262:416-25.

50. Alverdy JC. Microbiome medicine: this changes everything. J Am Coll Surg 2018;226:719-29.

51. Lobo DN, Bostock KA, Neal KR, Perkins PAC, Rowlands PBJ, Allison PSP. Effect of salt and water balance on recovery of gastrointestinal functions after elective colonic resection: a randomized controlled trial. Lancet 2002;359:1812-18.

52. MacKay G, Fearon K, McConnachie A, Serpell MG, Molloy RG, O’Dwyer PJ. Randomized clinical trial of the effect of postoperarive intravenous fluid restriction on recovery after elective colorectal surgery. Br J Surg 2006;93:1469-74.

53. Gan TJ, Soppitt A, Maroof M, el-Moalem H, Robertson KM, Moretti E, Dwane P, Glass PS. Goal-directed intraoperative fluid administration reduces length of hospital stay after major surgery. Anesthesiology 2002;97:280-6.

54. Walsh SR, Tang T, Bass S, Gaunt ME. Doppler guided intra-operative fluid management during major abdominal surgery: systematic review and meta-analysis. Int J Clin Pract 2008;62:466-70.

55. Myles PS, Bellomo R, Corcoran T, Forbes A, Peyton P, Story D, Christophi C, Leslie K, McGuinness S, Parke R, Serpell J, Chan MTV Painter T, McCluskey S, Minto G, Wallace S; Australian and New Zealand College of Anaesthetists Clinical Trials Network and the Australian and New Zealand Intensive Care Society Clinical Trials Group. Restrictive versus liberal fluid therapy for major abdominal surgery. N Engl J Med 2018;378:2263-74

56. Song F, Glenny AM. Antimicrobial prophylaxis in colorectal surgery: a systematic review of randomized controlled trials. Br J Surg 1998;85:1232-41

57. Nelson RL, Glenny AM, Song F. Antimicrobial prophylaxis for colorectal surgery. Cochrane Database Syst Rev 2009; doi: 
10.1002/14651858.CD001181.pub3.

58. Choi JW, Kim DK, Kin JK, Lee EJ, Kim JY. A retrospective analysis on the relationship between intraoperative hypothermia and postoperative ileus after laparoscopic colorectal surgery. PLoS One 2018;13:e0190711.

59. van der Pas MH, Haglind E, Cuesta MA, Fürst A, Lacy AM, Hop WC, Bonjer HJ; COlorectal cancer Laparoscopic or Open Resection II (COLOR II) Study Group. Laparoscopic versus open surgery for rectal cancer (COLOR II): short term outcomes of a randomized, phase 3 trial. Lancet Oncol 2013;14:210-8.

60. Bonjer HJ, Deijen CL, Abis GA, Cuesta MA, van der Pas MH, de Lange-de Klerk ES, Lacy AM, Bemelman WA, Andersson J, Angenete E, Rosenberg J, Fuerst A, Haglind E; COLOR II Study Group. A randomized trial of laparoscopic versus open surgery for rectal cancer. N Engl J Med 2015;372:1324-32.

61. Jesus EC, Karliczek A, Matos D, Castro AA, Atallah AN. Prophylactic anastomotic drainage for colorectal surgery. Cochrane Database Syst Rev 2004; doi: 10.1002/14651858.CD002100.pub2.

62. Bretagnol F, Slim K, Faucheron JL. Anterior resection with low colorectal anastomosis. To drain or not? Ann Chir 2005;130:336-9.

63. Peeters KC, Tollenaar RA, Marijnen CA, Klein Kranenbarg E, Steup WH, Wiggers T, Rutten HJ, van de Velde CJ; Dutch Colorectal Cancer Group. Risk factors for anastomotic failure after total mesorectal excision of rectal cancer. Br J Surg 2005;92:211-6.

64. Rondelli F, Bugiantella W, Vedovati MC, Balzarotti R, Avenia N, Mariani E, Agnelli G, Becattini C. To drain or not to drain extraperitoneal colorectal anastomosis? A systematic review and meta-analysis. Colorectal Dis 2014;16:35-42.

65. Denost Q, Rouanet P, Faucheron JL, Panis Y, Meunier B, Cotte E, Meurette G, Kirzin S, Sabbagh C, Loriau J, Benoist S, Mariette C, Sielezneff I, Lelong B, Mauvais F, Romain B, Barussaud ML, Germain C, Picat MQ, Rullier E, Laurent C; French Research Group of Rectal Cancer Surgery (GRECCAR). To drain or not to drain infraperitoneal anastomosis after rectal excision for cancer: the GRECCAR 5 randomized trial. Ann Surg 2017;265:474-80.

66. Nelson R, Edwards S, Tse B. Prophylactic nasogastric decompression after abdominal surgery. Cochrane Database Syst Rev 2007; doi: 10.1002/14651858.CD004929.pub3.

67. Zmora O, Madbouly K, Tulchinsky H, Hussein A, Khaikin M. Urinary bladder catheter drainage following pelvic surgery -- is it necessary for that long? Dis Colon Rectum 2010;53:321-6.

68. Gelalis ID, Arnaoutoglou CM, Politis AN, Batzaleksis NA, Katonis PG, Xenakis TA. Bacterial wound contamination during simple and complex spinal procedures. A prospective clinical study. Spine J 2011;11:1042-8.

69. Krezalek MA, Hyoju S, Zaborin A, Okafor E, Chandrasekar L, Bindokas V, Guyton K, Montgomery CP, Daum RS, Zaborina O, BoyleVavra S, Alverdy JC. Can methicillin-resistant staphylococcus aureus silently travel from the gut to the wound and cause postoperative infection? Modeling the "Trojan Horse Hypothesis". Ann Surg 2018;267:749-58.

70. Cohen I Jr, Rives JD. Antibiotic protection of colon anastomoses. Ann Surg 1955;141:707-17.

71. Clarke JS, Condon RE, Bartlett JG, Gorbach SL, Nichols RL, Ochi S. Preoperative oral antibiotics reduce septic complications of colon operations: results of a prospective, randomized, double-blind clinical study. Ann Surg 1977;186:251-9.

72. Tanner J, Padley W, Assadian O, Leaper D, Kiernan M, Edmiston C. Do surgical care bundles reduce the risk of surgical site infections in patients undergoing colorectal surgery? A systematic review and cohort meta-analysis of 8,515 patients. Surgery 2015;158:66-77.

73. McEvoy MD, Scott MJ, Gordon DB, Grant SA, Thacker JKM, Wu CL, Gan TJ, Mythen MG, Shaw AD, Miller TE. American society for enhanced recovery (ASER) and perioperative quality initiative (POQI) joint consensus statement on optimal analgesia within an enhanced recovery pathway for colorectal surgery: part1 - from the preoperative period to PACU. Perioper Med 2017;6:8.

74. Maher DP, White PF. Proposed mechanisms for association between opioid usage and cancer recurrence after surgery. J Clin Anest 2016;28:36-40.

75. Jørgensen H, Wetterslev J, Møiniche S, Dahl JB. Epidural local anaesthetics versus opioid-based analgesic regimens for postoperative gastrointestinal paralysis, PONV and pain after abdominal surgery. Cochrane Database Syst Rev 2000; doi: 10.1002/14651858. CD001893.

76. Kehlet H. Epidural analgesia and postoperative outcome. Lancet 2008;372:2109.

77. Merchea A, Lovely JK, Jacob AK, Colibaseanu DT, Kelley SR, Mathis KL, Spears GM, Huebner M, Larson DW. Efficacy and outcomes of intrathecal analgesia as part of an enhanced recovery pathway in colon and rectal surgical patients. Surg Res Pract 2018; doi: $10.1155 / 2018 / 8174579$.

78. Levy BF, Tilney HS, Downson HM, Rockall TA. A systematic review of postoperative analgesia following laparoscopic colorectal surgery. Colorect Dis 2010;12:5-15.

79. Jibril F, Sharaby S, Mohamed A, Wilby KJ. Intravenous versus oral acetaminophen for pain: systematic review of current evidence to support clinical decision-making. Can J Hosp Pharm 2015;68:238-47.

80. Bhangu A, Singh P, Fitzgerald JE, Slesser A, Tekkis P. Postoperative nonsgteroidal anti-inflammatory drugs and risk of anastomotic leak: meta-analysis of clinical and experimental studies. World J Surg 2014;38:2247-57.

81. Modasi A, Pace D, Godwin M, Smith C, Curtis B. NSAID administration post colorectal surgery increases anastomotic leak rate: systematic review/meta-analysis. Surg Endosc 2018; doi: 10.1007/s00464-018-6355-1.

82. McEvoy MD, Wanderer JP, King AB, Geiger TM, Tiwari V, Terekhov M, Ehrenfeld JM, Furman WR, Lee LA, Sandberg WS. A perioperative consult service results in reduction in cost and length of stay for colorectal surgical patients: evidence from a healthcare redesign project. Perioper Med (Lond) 2016;5:3.

83. Lloyd GM, Kirby R, Hemingway DM, Keane FB, Miller AS, Neary P. The RAPID protocol enhances patient recovery after both laparoscopic and open colorectal resections. Surg Endosc 2010;24:1434-9.

84. Wang L, Johnston B, Kaushal A, Cheng D, Zhu F, Martin J. Ketamine added to morphine or hydromorphone patient-controlled analgesia for acute postoperative pain in adults: a systematic review and meta-analysis of randomized trials. Can J Anaesth 2016;63:311-25.

85. Guo BL, Lin Y, Hu W, Zhen CX, Bao-Cheng Z, Wu HH, Kaye AD, Duan JH, Qu Y. Effects of systemic magnesium on post-operative analgesia: is the current evidence strong enough? Pain Physician 2015;18:405-18. 
86. Waldron NH, Jones CA, Gan TJ, Allen TK, Habib AS. Impact of perioperative dexamethasone on postoperative analgesia and sideeffects: systematic review and meta-analysis. Br J Anaesth 2013;110:191-200.

87. Goll V, Akça O, Greif R, Freitag H, Arkiliç CF, Scheck T, Zoeggeler A, Kurz A, Krieger G, Lenhardt R, Sessler DI. Ondansetron is no more effective than supplemental intraoperative oxygen for prevention of postoperative nausea and vomiting. Anesth Analg 2001;92:112-7.

88. Habib AS, White WD, Eubanks S, Pappas TN, Gan TJ. A randomized comparison of a multimodal management strategy versus combination antiemetics for the prevention of postoperative nausea and vomiting. Anest Analg 2004;99:77-81.

89. Funder JA, Tolstrup R, Jepsen BN, Iversen LH. Postoperative paralytic ileus remains a problem following surgery for advanced pelvic cancers. J Surg Res 2017;218:167-73.

90. Lee L, Liberman S, Charlebois P, Stein B, Kaneva P, Carli F, Feldman LS. The impact of complications after elective colorectal resection within an enhanced recovery pathway. Tech Colproctol 2018;22:191-9.

91. Sugawara K, Kawaguchi Y, Nomura Y, Suka Y, Kawasaki K, Uemura Y, Koike D, Nagai M, Furuya T, Tanaka N. Perioperative factors predicting prolonged postoperative ileus after major abdominal surgery. J Gastrointest Surg 2018;22:508-15.

92. Grass F, Slieker J, Jurt J, Kummer A, Solà J, Hahnloser D, Demartines N, Hübner M. Postoperative ileus in an enhanced recovery pathway - a retrospective cohort study. Int J Colorectal Dis 2017;32:675-81.

93. Pohl JM, Gutweiler S, Thiebes S, Volke JK, Klein-Hitpass L, Zwanziger D, Gunzer M, Jung S, Agace WW, Kurts C, Engel DR. Irf4dependent $\mathrm{CD} 103+\mathrm{CD} 11 \mathrm{~b}+$ dendritic cells and the intestinal microbiome regulate monocyte and macrophage activation and intestinal peristalsis in postoperative ileus. Gut 2017;66:2110-20.

94. Engel DR, Koscielny A, Wehner S, Maurer J, Schiwon M, Franken L, Schumak B, Limmer A, Sparwasser T, Hirner A, Knolle PA, Kalff JC, Kurts C. T helper type 1 memory cells disseminate postoperative ileus over the entire intestinal tract. Nat Med 2010;16:1407-13.

95. Grass F, Slieker J, Jurt J, Kummer A, Solà J, Hahnloser D, Demartines N, Hübner M. Postoperative ileus in an enhanced recovery pathway - a retrospective cohort study. Int J Colorectal Dis 2017;32:675-81.

96. Rohatiner T, Wend J, Rhodes S, Murrell Z, Berel D, Fleshner P. A prospective single-institution evaluation of current practices of early postoperative feeding after elective intestinal surgery. Am Surg 2012;78:1147-50.

97. Viscusi ER, Gan TJ, Leslie JB, Foss JF, Talon MD, Du W, Owens G. Peripherally acting mu-opioid recepton antagonists and postoperative ileus: mechanisms of action and clinical applicability. Anesth Analg 2009;108:1811-22.

98. Schwenk ES, Grant AE, Torjman MC, McNulty SE, Baratta JL, Viscusi ER. The efficacy of peripheral opioid antagonists in opioidinduced constipation and postoperative ileus: a systematic review of the Literature. Reg Anesth Pain Med 2017;42:767-77.

99. Creamer F, Balfour A, Nimmo S, Foo I, Norrie JD, Williams LJ, Fearon KC, Paterson HM. Randomized open-label phase II study comparing oxycodone-naloxone with oxycodone in early return of gastrointestinal function after laparoscopic colorectal surgery. Br J Surg 2017;104:42-51.

100. Purkayastha S, Tilney HS, Darzi AW, Tekkis PP. Meta-analysis of randomized studies evaluating chewing gum to enhance postoperative recovery following colectomy. Arch Surg 2008;143:788-93.

101. Short V, Herbert G, Perry R, Atkinson C, Ness AR, Penfold C, Thomas S, Andersen HK, Lewis SJ. Chewing gum for postoperative recovery of gastrointestinal function. Cochrane Database Syst Rev 2015; doi: 10.1002/ 14651858.CD006506.pub3.

102. Liu Q, Jiang H, Xu D, Jin J. Effect of gum ghewing on ameliorating ileus following colorectal surgery: a meta-analysis of 18 randomised controlled trials. Int J Surg 2017;47:107-15.

103. Atkinson C, Penfold CM, Ness AR, Longman RJ, Thomas SJ, Hollingworth W, Kandiyali R, Leary SD, Lewis SJ. Randomised clinical trial of postoperative chewing gum versus standard care after colorectal resection. Br J Surg 2016;103:962-70.

104. Hansen CT, Sørensen M, Møller C, Ottesen B, Kehlet H. Effect of laxatives on gastrointestinal functional recovery in fast-track hysterectomy: a double blind, placebo-controlled randomized study. Am J Obstetr Gynecol 2007;196:e1-7.

105. Martos-Benítez FD1, Gutiérrez-Noyola A2, Soto-García A2, González-Martínez I3, Betancourt-Plaza I. Program of gastrointestinal rehabilitation and early postoperative enteral nutrition: a prospective study. Updates Surg 2018;70:105-12.

106. Probst P, Ohmann S, Klaiber U, Hüttner FJ, Billeter AT, Ulrich A, Büchler MW, Diener MK. Meta-analysis of immunonutrition in major abdominal surgery. Br J Surg 2017;104:1594-1608.

107. Xu J, Sun X, Xin Q, Cheng Y, Zhan Z, Zhang J, Wu J. Effect of immunonutrition on colorectal cancer patients undergoing surgery: a meta-analysis. Int J Colorect Dis 2018;33:273-83.

108. Short V, Atkinson C, Ness AR, Thomas S, Burden S, Sutton E. Patient experiences of perioperative nutrition within an enhanced recovery after surgery programme for colorectal surgery: a qualitative study. Colorectal Dis 2016;18:O74-80.

109. Rinninella E, Persiani R, D’Ugo D, Pennestrì F, Cicchetti A, Di Brino E, Cintoni M, Miggiano GAD, Gasbarrini A, Mele MC. NutriCatt protocol in the enhanced recovery after surgery (ERAS) program for colorectal surgery: the nutritional support improves clinical and cost-effectiveness outcomes. Nutrition 2018;50:74-81.

110. Beloeil H, Slim K; Francophone Group of enhanced recovery after surgery. Sustainability of anaesthesia components of an enhanced recovery program (ERP) in colorectal and orthopaedics surgery. Anaesth Crit Care Pain Med 2018; doi: 10.1016/j.accpm.2018.01.005.

111. Veziant J, Leonard D, Pereira B, Slim K; French speaking Group for Enhanced Recovery after Surgery (GRACE). How does the application of surgical components in enhanced recovery programs for colorectal surgery change over time? Surgeon 2018; doi: 10.1016/j.surge.2018.03.003.

112. Balvardi S, Pecorelli N, Castelino T, Niculiseanu P, Liberman AS, Charlebois P, Stein B, Carli F, Mayo NE, Feldman LS, Fiore JF Jr. Measuring in-hospital recovery after colorectal surgery within a well-established enhanced recovery pathway: a comparison between hospital length of stay and time to readiness for discharge. Dis Colon Rectum 2018;61:854-60.

113. Grant MC, Pio Roda CM, Canner JK, Sommer P, Galante D, Hobson D, Gearhart S, Wu CL, Wick E. The impact of anesthesiainfluenced process measure compliance on length of stay: results from an enhanced recovery after surgery for colorectal surgery cohort. Anesth Analg 2018; doi: 10.1213/ANE.0000000000003458. 
114. Braga M, Scatizzi M, Borghi F, Missana G, Radrizzani D, Gemma M; Perioperative Italian Society. Identification of core items in the enhanced recovery pathway. Clin Nutr ESPEN 2018;25:139-44.

115. Jung AD, Dhar VK, Hoehn RS, Atkinson SJ, Johnson BL, Rice T, Snyder JR, Rafferty JF, Edwards MJ, Paquette IM. Enhanced recovery after colorectal surgery: can we afford not to use it? J Am Coll Surg 2018;226:586-93. 